\title{
Arañas y carábidos como potenciales bioindicadores en ambientes con distinto grado de intervención antrópica en el este uruguayo: un estudio preliminar
}

\section{Spiders and carabids as potential bioindicators in Eastern Uruguayan environments with different degree of anthropic intervention: a preliminary study}

Castiglioni, Enrique (1); García, Luis F. (2); Burla, Juan P. (1); Arbulo, Natalia (1); Fagúndez, César (1).

(1) Centro Universitario Regional del Este (CURE), Universidad de la República (UdelaR), Rocha, Uruguay.

(2) Centro Universitario Regional del Este (CURE), Universidad de la República (UdelaR), Treinta y Tres, Uruguay.

Contacto: bbcastbb@gmail.com

RECIBIDO: $31 / 3 / 2017$ - APROBADO: $30 / 6 / 2017$

\begin{abstract}
Resumen
Algunos grupos de artrópodos son considerados buenos indicadores de calidad ambiental. Para ello deben cumplir con algunas condiciones, como poseer características biológicas y morfológicas que faciliten su hallazgo e identificación. Las trampas de caída (pitfall) son ampliamente utilizadas para recolectar artrópodos predadores potencialmente bioindicadores de las características del ambiente que ocupan. Se realizaron recolecciones quincenales, durante nueve meses, en tres sistemas productivos con diferente grado de intervención antrópica en la cuenca de la Laguna Negra, Rocha: área con baja intensidad de pastoreo de vacunos; área con bajo pastoreo de ganado vacuno y ovino, y área con altas cargas de ganado vacuno y agricultura inverno-estival. En cada área se instalaron dos series de 10 trampas pitfall separadas a una distancia mínima de $100 \mathrm{~m}$ y a una distancia de 10 $\mathrm{m}$ entre trampas. Dos morfoespecies de arañas, Mesabolivar sp (Pholcidae) y Steatoda sp (Theridiidae), fueron determinadas como indicadoras de los ambientes con menor y mayor intensidad de disturbio, respectivamente. Otras cuatro morfoespecies, pertenecientes a las familias Nemesiidae, Oxyopidae, Lycosidae y Palpimanidae, fueron caracterizadas como detectoras de los diferentes ambientes. Cuatro morfoespecies de coleópteros de la familia Carabidae (Calosoma retusum, Galerita collaris, Brachinus sp y Notiobia sp) resultaron indicadoras del ambiente de mayor intensidad productiva, con agricultura.

Palabras clave: Predadores, Carabidae, Araneae, intensificación.
\end{abstract}

\begin{abstract}
Some arthropod groups are considered to be good bioindicators of environmental quality. For that, they must fill some conditions, such as biological and morphological characteristics that lead to their easy find and identification. The pit-fall traps are widely used as an efficient method to collect predator arthropods that may have a good potential as bioindicators of the characteristics of their environment. During a nine months period, fortnightly collects were made in three production systems with different intensity of anthropic intervention, in the Laguna Negra basin, Rocha, Uruguay: natural grassland with low intensity of bovine cattle grazing; bovine and sheep grazed area, and an area under high bovine grazing intensity with winter-summer agriculture. In each of the three areas two series of 10 pitfall traps were installed with a distance $100 \mathrm{~m}$ minimum between series and $10 \mathrm{~m}$ between traps. Two spider morphospecies, Mesabolivar sp (Pholcidae) y Steatoda sp (Theridiidae) appeared to be good indicators for the less and most intensified environments, respectively. Other four morphospecies of the families Nemesiidae, Oxyopidae, Lycosidae y Palpimanidae were characterized as detectors to different environments. Four morphospecies of the coleopteran family Carabidae (Calosoma retusum, Galerita collaris, Brachinus sp and Notiobia sp) were defined as indicators of the more intensified system, with agriculture.

Keywords: Predators, Carabidae, Araneae, intensification.
\end{abstract}




\section{Introducción}

La pérdida de la biodiversidad, como elemento clave de funcionamiento y estabilidad de los ecosistemas, acarrea una reducción en la eficiencia con que las comunidades ecológicas capturan recursos, producen biomasa y descomponen y reciclan nutrientes biológicamente esenciales (Cardinale, et al., 2012). Estas deficiencias de funcionamiento debidas a la pérdida de la heterogeneidad original en los agroecosistemas redundan en una disminución de los niveles de servicios que los ecosistemas brindan al ser humano (Naeem, et al., 1999).

A nivel global, el monitoreo es necesario para comparar tendencias causadas por el incremento en la homogeneidad de los paisajes, y constituye una retroalimentación indispensable entre las acciones humanas y el ambiente. Mediante el monitoreo se puede determinar el estatus de la diversidad biológica a uno o más niveles ecológicos, evaluar cambios en el tiempo y el espacio y marcar líneas de acción para decisiones de manejo en términos de producción y conservación (Moreno, 2001).

Para establecer las condiciones actuales de los ecosistemas o predecir las futuras se recurre al uso de indicadores relativamente simples y fáciles de interpretar que permiten aislar aspectos clave del ambiente de un conjunto abrumador de señales. Los bioindicadores que reflejan el estado abiótico y biótico de un ambiente son ampliamente utilizados para detectar, cuantificar y monitorear impactos ambientales, incluidas las perturbaciones debidas a las actividades antropogénicas (Rainio y Niemelä, 2003; Niemi y McDonald, 2004).

Muchos artrópodos, por el hecho de ser sensibles a los cambios en el ambiente, particularmente a los que resultan de las actividades antrópicas, son usados como indicadores de biodiversidad (Rocha, et al., 2010; Cameron y Leather, 2012). Sus respuestas rápidas a los cambios ambientales, su pequeño tamaño, su corta vida, las altas tasas reproductivas y las abundancias de muchas especies permiten el ajuste de sus poblaciones a las condiciones cambiantes, incluyendo las modificaciones en el manejo de los suelos y los cambios del paisaje. Por ello, muchos artrópodos funcionan como herramientas para el monitoreo de la calidad del ambiente y los efectos del cambio a lo largo del tiempo y el espacio (Kremen, et al., 1993; Schowalter, 2006).

Uno de los objetivos principales para encontrar posibles bioindicadores es identificar especies u otras unidades taxonómicas que puedan representar de modo realista los disturbios en el ambiente y reflejar las respuestas de otras especies o de la biodiversidad en su conjunto (Rainio y Niemelä, 2003).

Entre los usos posibles de estos organismos se incluyen las estimaciones de la riqueza específica de una zona, la caracterización y seguimiento de ecosistemas, desde un enfoque conservacionista y la detección de alteraciones de los ecosistemas, que son difíciles de medir y cuantificar de un modo directo (Ribera y Foster, 1997). La información obtenida a partir de los bioindicadores puede ser usada para predecir cambios en el ambiente, identificar acciones para su remediación, o identificar cambios o tendencias en los propios indicadores (Niemi y McDonald, 2004).

Además de su rol como predadores de artrópodos fitófagos, las arañas (Arachnida, Araneae) han sido citadas como adecuadas para representar la diversidad remanente a diversas escalas espaciales (Gaspar, et al., 2010). En algunos estudios se encontró que la composición de arañas en especies o grupos funcionales difiere en función del grado de intervención antrópica o de la estructura vegetal dominante (Chen y Tso,
2004; Collins, et al., 1996; Schmidt, et al., 2005). Aunque su rol como indicadores ha sido probado en áreas forestales (Simó, et al., 2011) o ambientes costeros (Ghione, et al., 2013), el uso de este grupo como bioindicador aún es limitado en Uruguay.

Los carábidos (Coleoptera, Carabidae) y otras familias de coleópteros han sido citados como buenos indicadores de la diversidad del ambiente, aunque su valor está influenciado por el método de muestreo y la escala espacial del estudio (Sauberer, et al., 2004; Duelli y Obrist, 1988; 2003).

La región este de Uruguay presenta características únicas que son útiles para analizar los efectos de los cambios en el uso de la tierra sobre la diversidad. Siguiendo la tendencia nacional de los cambios recientes, la proporción de suelo destinada a la forestación y a la agricultura intensiva ha aumentado, sustituyendo principalmente pastizales naturales que sustentan diversos sistemas ganaderos.

En el marco del Convenio sobre la Diversidad Biológica (CBD), Uruguay presenta estrategias y planes de acción nacionales para la conservación y uso sostenible de la diversidad biológica, implementados a través de diversas herramientas como leyes, programas y proyectos (DINAMA, 2010). En el departamento de Rocha se sitúan cuatro de las ocho áreas protegidas del Sistema Nacional de Áreas Protegidas (SNAP) y un área de manejo de hábitats y/o especies compartido con Maldonado (Laguna Garzón). El SNAP cuenta, además, con otras ocho áreas que están en proceso de ingreso y cuatro propuestas de ingreso en evaluación (MVOTMA, 2015). En algunas de ellas, como en el Paisaje Protegido Laguna de Rocha, existen planes de manejo que regulan, entre otras, las actividades productivas permitidas. La diversidad de paisajes y el gradiente de intensidad de uso de la tierra resultan muy útiles a la hora de seleccionar, evaluar y validar taxones como potenciales bioindicadores.

El uso de organismos indicadores, no obstante, requiere conocimiento de la taxonomía del grupo y métodos disponibles para la facilidad de recolección. Entre estos, las trampas pasivas de caída tipo pitfall han sido ampliamente utilizadas en estudios de biodiversidad con artrópodos de suelo (Buddle y Hammond, 2003), a pesar de los sesgos que puede tener este método (Curtis, 1980). Entre otros factores, inciden el tamaño de las trampas, los artrópodos objetivos, el empleo o no de líquido fijador, sus componentes y proporciones. Según Lange et al. (2011), el número de especies de carábidos recolectados no es afectado significativamente por el aumento del diámetro de las trampas (entre 4,5 y 9,0 cm), aunque se captura un número mayor de ejemplares en las trampas más grandes. Los autores recomiendan trampas con diámetro no menor de $9 \mathrm{~cm}$ de diámetro para una recolección más eficiente de los artrópodos epígeos. Work et al. (2002), contrariamente, recomiendan aumentar el número de trampas de menor diámetro (en un rango de 4,5 a $20 \mathrm{~cm}$ ) como medida de incrementar la eficiencia de caracterización de estos organismos y reducir la tasa de captura de vertebrados no blanco. Brennan et al. (1999) demostraron una correlación directa entre el diámetro de la trampa y la eficiencia de captura de arañas. La eficiencia de captura de arañas se incrementó con la incorporación de barreras de 4 a 6 metros de largo a las trampas de $11,1 \mathrm{~cm}$ de diámetro (Brennan, et al., 2005). Sin embargo, Winder et al. (2001) indicaron que la eficiencia de las trampas con barreras aplicaba solo para ciertos grupos de arañas. Adicionalmente, este método incrementa la captura de organismos no blanco, como vertebrados (Bury y Corn, 1987). El número de trampas y el empleo o no de agentes fijadores también ha sido discutido 
para evaluar la eficiencia de captura de carábidos y arañas (Winder, et al., 2001; Schmidt et al., 2006). El líquido fijador puede afectar la eficiencia de captura para estos grupos y sus componentes tienen incidencia en la preservación de la muestra. El monoetilenglicol demostró ser efectivo como solvente en la captura tanto de arañas como carábidos (Holopainen, 1992; Weeks y McIntyre, 1997; Schmidt, et al., 2006; Jud y Schmidt-Entling, 2008).

Este estudio tiene como objetivo principal la búsqueda de arácnidos y carábidos con potencial valor como bioindicadores de biodiversidad y calidad ambiental en tres áreas con diferentes formaciones vegetales y con diferente grado de transformación en el uso del suelo. Se espera contribuir al potencial monitoreo de organismos capaces de detectar y predecir cambios en el ambiente de forma sencilla y de bajo costo para su incorporación en planes de manejo de sistemas productivos sostenibles, de áreas protegidas y para la conservación de la biodiversidad en general.

\section{Materiales y Métodos}

Se realizaron recolectas quincenales con trampas pasivas de caída (pitfall), protegidas con una estructura de hierro para evitar el daño del ganado y con techo para evitar el sobrellenado de los recipientes de captura con agua de lluvia (Figura 1).

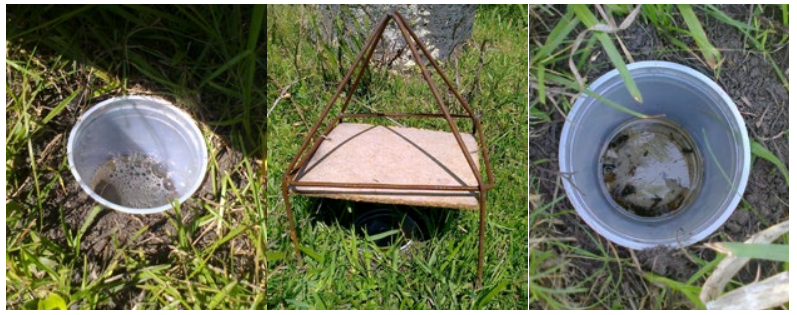

Figura 1. Trampas de caída (pitfall) (A); con protección de hierro y techo $(\mathrm{B})$; con la recolección interior $(\mathrm{C})$.
La toma de datos se realizó entre diciembre de 2014 y agosto de 2015, en tres sitios de muestreo localizados en las cercanías de la ciudad de Castillos, cuenca de la Laguna Negra, departamento de Rocha, Uruguay (Figura 2). Se definieron tres áreas con un gradiente de intensidad de uso del suelo: a) área de pastizal natural (CN: campo natural) sobre la costa de la Laguna Negra, con acceso sobre el km 21,500 de la Ruta 16 y con baja intensidad de pastoreo con vacunos; b) área de pastizal bajo pastoreo de ganado vacuno y lanar (CG: campo ganadero), con acceso por camino lateral desde $\mathrm{km} 23$ de Ruta 16 ; c) sistema integrado de producción ganadera intensiva con agricultura inverno-estival (AG: agrícola-ganadero), establecimiento sito en $\mathrm{km} 30$ de Ruta 16.

Las áreas se seleccionaron dentro de una matriz de pastizal nativo, con similares condiciones ambientales originales y climáticas, con una separación mayor a $1 \mathrm{~km}$ entre sí, buscando que las comunidades de artrópodos capturados fueran independientes.

En cada una de las áreas se colocaron dos series de diez trampas pitfall, con una distancia mínima de $100 \mathrm{~m}$ entre las series y separadas $10 \mathrm{~m}$ entre sí. Cada trampa está formada por dos recipientes de poliestireno, de $11,6 \mathrm{~cm}$ de altura y diámetro superior de $11 \mathrm{~cm}$ e inferior de $9 \mathrm{~cm}$ (Volumen $=1 \mathrm{~L})$. El recipiente exterior se perforó en su base y se dejó dentro del suelo durante todo el período de muestreo. El segundo recipiente (de captura) se introdujo en el exterior perforado, con un volumen aproximado al $20 \%$ del total de una solución fijadora de monoetilenglicol $(0,6 \mathrm{~L})$, ácido acético $(100 \mathrm{~mL})$, detergente $(50$ $\mathrm{mL}$ ) y agua corriente (hasta completar $5 \mathrm{~L}$ ). La recuperación de las recolectas y el reemplazo del líquido fijador se realizó quincenalmente. La utilización de los dobles recipientes facilita y agiliza la tarea de recuperación de muestras y el recambio de la solución fijadora, y evita el intercambio de gases del suelo que ocurre en ese procedimiento si los recipientes están en contacto con el sustrato (Schirmel, et al., 2010).

La presencia de las especies vegetales se registró en torno a las series de trampas de las diferentes áreas, utilizando un tiempo de búsqueda de $30 \mathrm{~min} /$ hombre por estación de

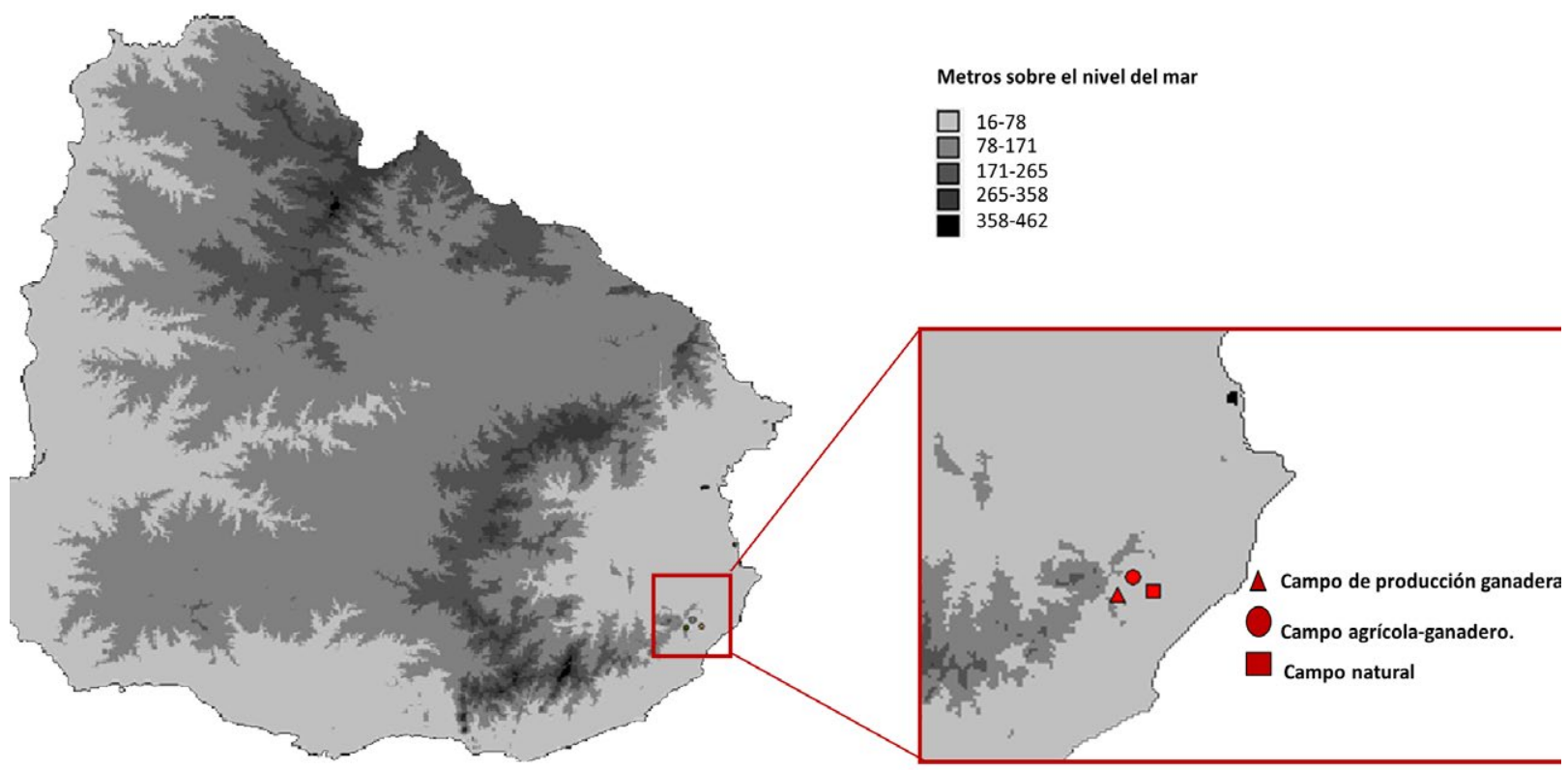

Figura 2. Localización de los ambientes estudiados en la cuenca de la Laguna Negra, Rocha, Uruguay. 
muestreo. Las especies se agruparon en diferentes categorías según la forma de vida registrada en cada zona (Tabla 1). Este sistema se basa en la ubicación y posición de las yemas vegetativas, caracteres adaptativos de los que dependen el crecimiento, desarrollo y reproducción de la especie, a posteriori de la estación adversa (Matteucci y Colma, 1982). Las variaciones en las formas de vida encontradas en cada área se graficaron como espectros biológicos (Matteucci y Colma, 1982) que imprimen una fisonomía determinada según su dominancia. Por ejemplo, el dominio de árboles define un bosque, el de arbustos un matorral y el de herbáceas un pastizal.

\begin{tabular}{|c|l|}
\hline Forma de vida & Definición \\
\hline Árbol & $\begin{array}{l}\text { Planta perenne, de raíz, tronco y } \\
\text { ramas leñosas, generalmente con } \\
\text { tronco único y un porte mayor a los 4 } \\
\text { metros de altura, según las condiciones } \\
\text { ambientales del lugar. }\end{array}$ \\
Arbusto & $\begin{array}{l}\text { Planta perenne, de raíz, tronco y ramas } \\
\text { leñosos, generalmente con varios } \\
\text { troncos y un porte menor a los 4 } \\
\text { metros de altura, según las condiciones } \\
\text { ambientales del lugar. }\end{array}$ \\
Epífita & $\begin{array}{l}\text { Planta generalmente perenne que crece } \\
\text { y se desarrolla sobre otra planta. }\end{array}$ \\
Suculenta & $\begin{array}{l}\text { Planta perenne, se incluye a la familia } \\
\text { de las Cactáceas. }\end{array}$ \\
Trepadora & $\begin{array}{l}\text { Planta perenne que crece apoyada/ } \\
\text { sostenida en otra planta. } \\
\text { Planta perenne, de base leñosa (parte } \\
\text { inferior) que le da una fisonomía entre } \\
\text { arbusto y hierba. } \\
\text { Planta sin leño. }\end{array}$ \\
Herbácea & \\
\hline
\end{tabular}

Tabla 1. Criterios utilizados para agrupar las diferentes formas de vida de las especies vegetales (adaptado de Raunkiaer, en Braun-Blanquet, 1964).

La asignación de las categorías de formas de vida para las especies vegetales registradas se realizó en el Laboratorio de Sistemática y Taxonomía, y la separación, catalogación, cuantificación e identificación de los carábidos y la separación de los arácnidos de las muestras tuvo lugar en el Laboratorio de Biología Animal, ambos en el Centro Universitario Regional del Este (CURE), sede Rocha. La catalogación, cuantificación e identificación de los arácnidos se realizó en el Laboratorio de Biología Animal del CURE, sede Treinta y Tres.

Los artrópodos recolectados fueron identificados a nivel de género o morfoespecie y agrupados a nivel de familia. Teniendo en cuenta el elevado número de familias dentro de los arácnidos, la abundancia se agrupó a nivel de orden. Las arañas fueron identificadas a nivel de familia utilizando la clave de Benamú (2007) y la determinación a nivel de género se llevó a cabo utilizando descripciones específicas para cada grupo o mediante la asesoría de especialistas. Los carábidos fueron identificados con ayuda de la clave de géneros de Reichardt (1977) y la revisión comparativa con los especímenes de las colecciones entomológicas de las Facultades de
Ciencias y Agronomía, Universidad de la República (UdelaR). Los ejemplares colectados serán utilizados para generar una colección de referencia en el CURE.

Para evaluar el potencial de las distintas morfoespecies como indicadoras se utilizó el índice de valor indicativo (IndVal), entre 0 (no indicativo) hasta $100 \%$ (indicador). Este índice ha sido ampliamente utilizado para evaluar el potencial de distintos grupos como bioindicadores y se apoya en los conceptos de especificidad y fidelidad basados en la abundancia e incidencia de un organismo en un ambiente o estado ecológico particular (Dufrêne y Legendre, 1997). Para la selección de las morfoespecies bioindicadoras se utilizaron los criterios descritos por Tonelli et al. (2017), de acuerdo a los valores significativos para el índice. Con base en esos criterios se categorizan las especies seleccionadas como indicadoras cuando su valor se sitúa entre $70-100 \%$ y como detectoras cuando el valor se sitúa entre el 45-70\%.

Los valores del índice del valor indicativo fueron analizados mediante el paquete estadístico Indicspecies (Dufrêne y Legendre, 1997) del software R versión 3.3.3 (R core Team, 2016). En los resultados solo se seleccionan las morfoespecies que presentan valores significativos para el índice $(\mathrm{p}<0.05)$.

\section{Resultados}

\section{Abundancia}

En el período considerado se recolectaron 6.530 ejemplares de arácnidos ubicados en 166 morfoespecies y cinco órdenes. El orden Araneae fue el más abundante, seguido por Acari, Opiliones, Scorpiones y Pseudoescorpiones (Figura 3). En los coleópteros (Insecta: Coleoptera) las dos familias más abundantes fueron Staphylinidae y Carabidae, cuyos integrantes son mayoritariamente predadores de otros insectos y artrópodos.

\section{Especies indicadoras}

En la familia Carabidae, cuatro morfoespecies de un total de 37 identificadas se determinaron como indicadoras (mspp 6c, 9c, 10c y 11c) para el sitio con mayor intensidad de intervención antrópica (AG) y dos morfoespecies detectoras para el sitio de bajo pastoreo intensivo con ganado (CG). Las morfoespecies de Carabidae Calosoma retusum (msp9c), Galerita collaris (msp10c), Brachinus sp. (msp6c) y Notiobia sp. (msp11c) resultaron indicadoras del sistema intensificado (AG).

En el total de arácnidos, solo las arañas presentaron taxones verificados como indicadores. Dentro de este orden, una morfoespecie (msp99; Mesabolivar sp.) de la familia Pholcidae fue la mejor indicadora del ambiente con menor intensidad de disturbio $(\mathrm{CN})$, mientras que una morfoespecie (msp13; Chaco sp.) de la familia Nemesiidae resultó detectora para este sitio (Tabla 2). Una morfoespecie (msp41; Steatoda sp.) de la familia Theridiidae fue la mejor indicadora del sitio AG, mientras que para el sitio CG únicamente se determinaron morfoespecies detectoras, pertenecientes a las familias $\mathrm{Ox}-$ yopidae (msp10; Oxyopes sp.), Lycosidae (msp30; Lycosa sp.) y Palpimanidae (msp62; Othiothops sp.). Las morfoespecies indicadoras se correspondieron con su abundancia en los diferentes ambientes (Figura 4). 


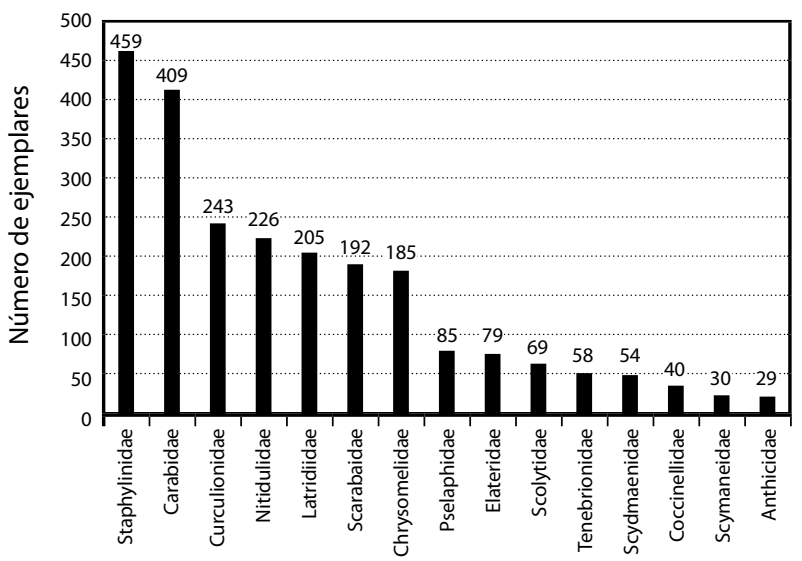

Familia

(B)

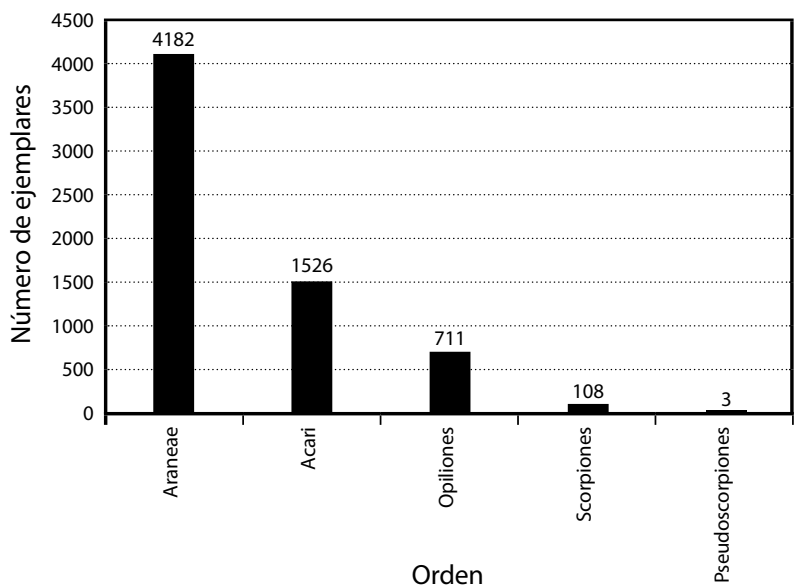

Figura 3. Abundancia de individuos de las 15 familias más abundantes de Coleoptera (A) y de los órdenes de Arachnida (B), en el total de las recolectas.

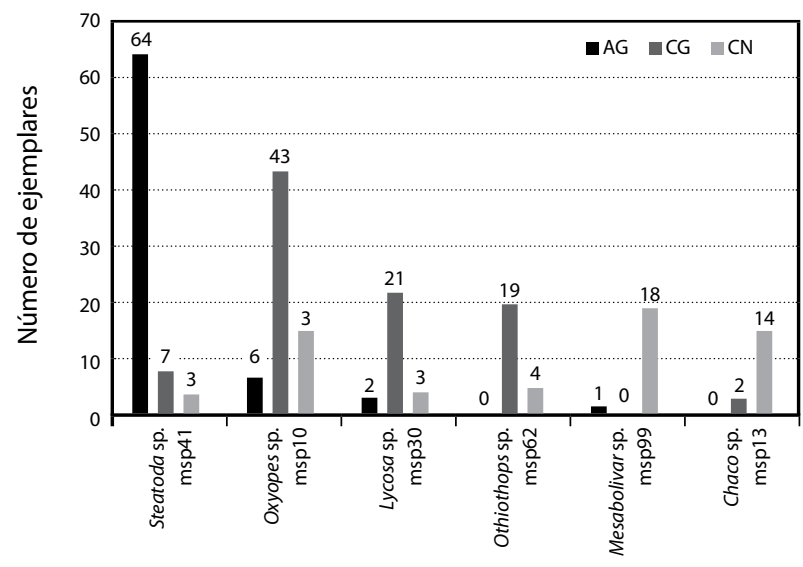

Morfoespecie

(B)

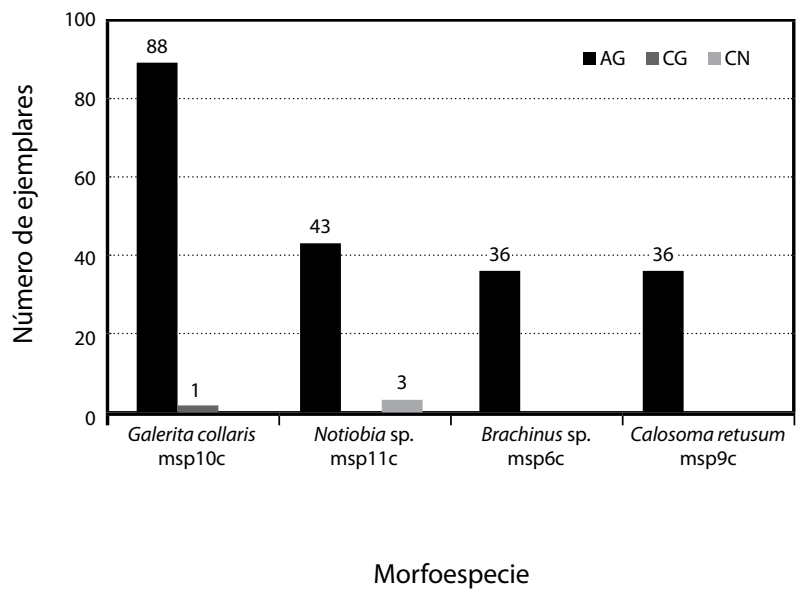

Figura 4. Abundancia de morfoespecies de carábidos (A) y arañas (B) según el sitio de recolección: agrícola-ganadero (AG), campo ganadero con alta intensidad de pastoreo (CG) y campo natural con baja intensidad de pastoreo $(\mathrm{CN})$.

\begin{tabular}{|c|c|c|c|c|c|c|}
\hline & \multirow{2}{*}{ Morfoespecie } & \multirow{2}{*}{ Familia } & \multirow{2}{*}{ Género } & \multicolumn{3}{|c|}{ Valor índice en cada sitio } \\
\hline & & & & AG & CG & $\mathrm{CN}$ \\
\hline \multirow{2}{*}{ Indicadoras } & msp41 & Theridiidae & Steatoda & 72,0 & & \\
\hline & msp99 & Pholcidae & Mesabolivar & & & 72,2 \\
\hline \multirow{4}{*}{ Detectoras } & msp10 & Oxyopidae & Oxyopes & & 64,0 & \\
\hline & msp30 & Lycosidae & $L y \cos a$ & & 60,3 & \\
\hline & msp62 & Palpimanidae & Othiothops & & 53,8 & \\
\hline & msp13 & Nemesiidae & Chaco & & & 51,2 \\
\hline
\end{tabular}

Tabla 2. Morfoespecies indicadoras y detectoras y su respectiva familia, de los sitios agrícola-ganadero (AG), ganadero con alta intensidad de pastoreo (CG) y campo natural con baja intensidad de pastoreo $(\mathrm{CN})$. 


\begin{tabular}{|c|c|c|c|}
\hline \multirow{2}{*}{ Sitio } & \multicolumn{2}{|c|}{ Ubicación } & \multirow{2}{*}{ Formaciones vegetales } \\
\cline { 2 - 3 } & Latitud & Longitud & Avena \\
\hline \multirow{2}{*}{ AG } & $34^{\circ} 02^{\prime} 32.00^{\prime \prime} S$ & $53^{\circ} 50^{\prime} 06.70^{\prime \prime} \mathrm{O}$ & Pastizal con cañada \\
\hline \multirow{2}{*}{$\mathrm{CG}$} & $34^{\circ} 02^{\prime} 31.61^{\prime \prime} \mathrm{S}$ & $53^{\circ} 50^{\prime} 05.15^{\prime} \mathrm{O}$ & Caraguatal con palmar \\
& $34^{\circ} 05^{\prime} 27.40^{\prime \prime} \mathrm{S}$ & $53^{\circ} 52^{\prime} 15.50^{\prime \prime} \mathrm{O}$ & Bosque serrano \\
& $34^{\circ} 05^{\prime} 27.71^{\prime \prime} \mathrm{S}$ & $53^{\circ} 52^{\prime} 13.73^{\prime} \mathrm{O}$ & Pastizal \\
\hline \multirow{2}{*}{$\mathrm{CN}$} & $34^{\circ} 05^{\prime} 28.00^{\prime \prime} \mathrm{S}$ & $53^{\circ} 52^{\prime} 14.10^{\prime \prime} \mathrm{O}$ & Pastizal \\
& $34^{\circ} 05^{\prime} 01.07^{\prime \prime} \mathrm{S}$ & $53^{\circ} 45^{\prime} 43.08^{\prime} \mathrm{O}$ & Bosque serrano \\
\hline
\end{tabular}

Tabla 3. Localización (grados, minutos y segundos) y formaciones vegetales registradas en junio de 2015 en las tres áreas de muestreo (AG, CG y CN).

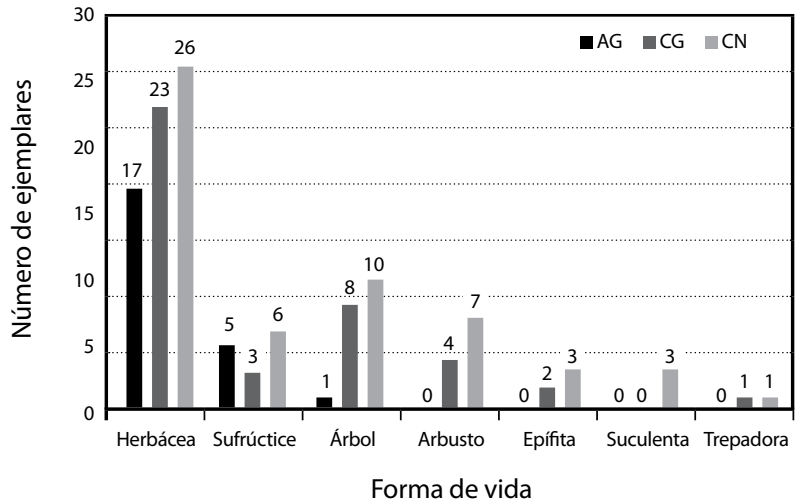

Figura 5. Número de especies agrupadas en las diferentes formas de vida de las especies vegetales registradas en los diferentes ambientes: área agrícola-ganadera (AG), campo ganadero, alta intensidad de pastoreo (CG), campo natural, baja intensidad de pastoreo $(\mathrm{CN})$.

\section{Caracterización de las formaciones vegetales}

Las tres áreas presentaron diferentes formaciones vegetales y formas de vida asociadas a una matriz de pastizales pertenecientes a la cuenca de la Laguna Negra. El área con un sistema agrícola ganadero (AG) presentaba un cultivo de avena para pastoreo, asociado a la formación vegetal pastizal con cañada (Tabla 3). Los sistemas sin agricultura (CG y CN) se caracterizaron por la dominancia de la formación vegetal pastizal, asociada a bosque serrano y a caraguatal con palmar, respectivamente.

Las proporciones de las diferentes formas de vida (espectros biológicos) muestran una mayor similitud en las variaciones de las formas de vida en las áreas ganaderas sin agricultura (CG y CN), en relación al sistema con mayor intensificación del uso del suelo (AG; ganadería y agricultura) (Figura 5).

\section{Discusión}

Los cambios en la abundancia de las especies o grupos indicadores bajo condiciones ecológicas distintas suelen ser notorios. Las especies o grupos detectores se relacionan con procesos ecológicos graduales a los que responden $-\mathrm{y}$ pueden ser evaluados- mediante cambios paulatinos en su abundancia (Tonelli, et al., 2017). Aunque los resultados pueden haber estado parcialmente sesgados por el uso de trampas pitfall como único método de muestreo, o inclusive por las características del líquido fijador empleado (Pekár, 2002), han demostrado ser más eficientes cuando se las compara con otras metodologías, en ambos grupos. Por ejemplo, Churchill y Arthur (1999) compararon la eficiencia de distintos métodos de muestreo para evaluar la riqueza de arañas en sistemas costeros de Tasmania. Estos autores encontraron que las trampas de caída reflejaban de manera más aproximada la riqueza total de especies en relación a otros métodos, colectando con aquellas $84 \%$ de especies y $94 \%$ de familias del total representado en la zona. En el caso de los carábidos, Skvarla y Dowling (2017) realizaron un estudio similar y, comparando distintos métodos de muestreo sobre distintos grupos de escarabajos, encontraron que las trampas pitfall fueron un método altamente efectivo, solas o en combinación con trampas Malaise.

Tradicionalmente, los carábidos han sido utilizados como indicadores de calidad ambiental, tanto de ambientes altamente conservados como de áreas con polución (Koivula, 2011). En este trabajo las cuatro morfoespecies destacadas como indicadoras se asociaron con el sistema agrícola-ganadero, ambiente de mayor grado de intervención antrópica (AG). Esta área está bajo una intensa acción de pastoreo intensivo de ganado en altas cargas, a la que se suma la perturbación de la actividad agrícola asociada al uso de insumos externos al sistema, particularmente fertilizantes y productos fitosanitarios. La presencia de estas morfoespecies de carábidos como indicadores de zonas perturbadas podría explicarse por una mayor tolerancia a las perturbaciones por intervención antrópica. Algunas especies de esta familia han sido citadas como especialistas de espacios abiertos o con vegetación alterada, lo que puede explicar su presencia en este tipo de ambientes (Niemelä, et al., 1993). Es posible, también, que pueda haber existido una atracción hacia la zona de cultivo por parte de los insectos fitófagos que constituyen sus presas. Este efecto ha sido verificado para predadores que se desplazan desde la vegetación circundante hacia el interior de los cultivos debido a la abundancia de presas (Bryan y Wratten, 1984).

Existen varios estudios que han demostrado el efecto negativo de las actividades antrópicas utilizando como 
modelos los arácnidos en Uruguay. Entre ellos se destacan principalmente ambientes urbanos o ecosistemas nativos (Capocasale y Gudynas, 1993; Costa, et al., 1991; Pérez-Miles, et al., 1999). Aunque el uso de las arañas como bioindicadores de perturbación en sistemas agrícolas se ha evaluado en otros ambientes como el mediterráneo (Barriga, et al., 2010), los estudios acerca de esta temática en sistemas agrícolas de Uruguay son escasos, con algunas investigaciones realizadas en sistemas forestales (Simó, et al., 2011).

El hecho de que las arañas hayan sido los únicos organismos bioindicadores dentro de los arácnidos fue inesperado, teniendo en cuenta que este rol ha sido citado también para ácaros, escorpiones, opiliones y pseudoescorpiones (Gerlach, et al., 2013). Posiblemente, la similitud de la matriz general de vegetación de pastizal y la proporción de las formas de vida vegetales presentes en las áreas evaluadas hayan resultado en determinar ambientes muy similares para la expresión de estos otros cuatro grupos. Esta hipótesis podría estar sustentada en las observaciones de Hore y Uniyal (2008) quienes verificaron en cinco diferentes tipos de vegetación en India que la composición de especies de arañas fue menos variable en los sitios con una vegetación similar que entre sitios con vegetación contrastante.

El caso de las morfoespecies de arañas de las familias Pholcidae y Nemesiidae, que fueron detectadas como indicadora y detectora para $\mathrm{CN}$, respectivamente, sugiere que tienen cierto grado de especificidad ambiental y poca tolerancia a cambios al asociarse con el ambiente de menor grado de intervención antrópica. Aunque las arañas de aquella familia suelen asociarse con ambientes antropizados, la presencia de algunas especies del género Mesabolivar se ha relacionado con determinadas estructuras vegetales o zonas húmedas o aledañas a cuerpos de agua que, en el caso del monte nativo, eran más frecuentes (Machado, et al., 2007; Machado, et al., 2013). Las arañas de la familia Nemesiidae, sin embargo, pueden haberse visto más afectadas por alteraciones ambientales como consecuencia de factores tales como su limitada capacidad de dispersión y su sensibilidad a cambios ambientales, características presentes, en general, en arañas migalomorfas (Ferreti, et al., 2014).

La caracterización de Oxiopus sp. como detectora en el campo ganadero (CG) podría estar explicada por su asociación con determinadas especies de la formación vegetal del sitio, como ha sido demostrado para algunas especies de la familia Oxyopidae, del género Peucetia (Vasconcellos-Neto, et al., 2007). A su vez, factores como la complejidad del paisaje y la presencia de rocas y otros posibles refugios podrían explicar el rol de detectoras de las morfoespecies de las familias Palpimanidae y Lycosidae. En el caso particular de esta última familia, la especie Allocosa brasiliensis ha sido sugerida como bioindicador de dunas costeras (Ghione, et al., 2013), por lo que es posible pensar en otras especies de Lycosidae como potenciales bioindicadoras. En contraste, la presencia de las arañas de la familia Theridiidae no es extraña en el sitio más antropizado (AG), debido a que las especies de esta familia son frecuentes en ambientes agrícolas (Alcayaga, et al., 2013).

Es importante destacar que los efectos de la intensificación en la riqueza de especies, cuando se comparan entre comunidades locales, muchas veces están afectados por la estructura del paisaje local (Duffey, 2012). La riqueza total de especies en los agroecosistemas a menudo está fuertemente influenciada por la proximidad de parches de vegetación nativa (semi-natural), como ha sido observado tanto para arañas (Hendrickx, et al., 2007) como para carábidos (Thomas y Marshall, 1999).
Al mismo tiempo, existen variaciones en la forma en que las diferentes especies reaccionan a las fuentes de recursos en ambientes contiguos como los cultivos agrícolas y las zonas herbáceas adyacentes (Fournier y Loreau, 1999).

De un modo general, independientemente de los factores del ambiente y de la biología de las predadores que intervienen en estas interrelaciones, cabe subrayar el rol beneficioso que cumplen estos organismos en el control de fitófagos plaga, por lo que la conservación de sus poblaciones debería ser el factor de prioridad en los agroecosistemas (Bolduc, et al., 2005). Por este motivo, además del rol que puedan desempeñar como indicadores, resultaría de gran interés estudiar de forma complementaria su función como enemigos naturales de las poblaciones de determinadas plagas de interés particular.

Considerando el rol de los carábidos y arácnidos en la disminución de las poblaciones de artrópodos nocivos para los ambientes productivos, su potencial como bioindicadores de la intensificación ambiental y las ventajas comparativas que presentan en relación a otros grupos de organismos utilizados (por ejemplo, vertebrados), es esperable que estos resultados contribuyan a la incorporación de estos artrópodos en programas de monitoreo del grado de disturbio en distintos ambientes, que en Uruguay aún son poco utilizados.

\section{Reconocimientos}

A los Sres. Walter Branaa, Fernando Ramos, Mario Cardoso, Ing. Agr. Agustín Piñeyrúa, por haber permitido el acceso a los sitios de estudio. Al Instituto Nacional de Investigación Agropecuaria (INIA-Uruguay), por el financiamiento del Proyecto FPTA No 312 en el marco de la Convocatoria 2012. Al Mag. Alvaro Laborda y al Dr. Fernando Pérez, de Facultad de Ciencias, por su colaboración en la identificación de las arañas. Al Dr. Enrique Morelli, de Facultad de Ciencias, por su apoyo en la identificación de Coleoptera.

\section{Referencias}

Alcayaga, O.E., Pizarro-Araya, J., Alfaro F.M. y CepedaPizarro, J., 2013. Arañas (Arachnida, Araneae) asociadas a agroecosistemas en el Valle de Elqui (Región de Coquimbo, Chile). En: Revista Colombiana de Entomología, 39, pp.150-154.

Barriga, J.C., Lassaletta, L. y Moreno, A.G., 2010. Groundliving spiderr assemblages from Mediterranean habitats under different management conditions. En: The Journal of Arachnology, 38, pp.258-269.

Benamú, M. A., 2007. Clave para la determinación de algunas familias de arañas (Araneae, Araneomorphae) del Uruguay. En: Boletín Sociedad Zoológica del Uruguay, 1, pp.1-19.

Bolduc, E., Buddle, C.M., Bostanian, N.J. y Vincent, C., 2005. Ground-dwelling spider fauna (Araneae) of two vineyards in Southern Quebec. En: Environmental Entomology, 34(3), pp.635-645.

Braun-Blanquet, J. 1979. Fitosociología. Bases para el estudio de las comunidades vegetales. Madrid: Blume.

Brennan, K.E.C., Majer, J.D. y Reygaert, N., 1999. Determination of an optimal pitfall trap size for sampling spiders in a western australian jarrah forest. En: Journal of Insect Conservation, 3, pp.297-307. 
Brennan, K.E.C., Majer, J.D. y Moir, M.L., 2005. Refining sampling protocols for inventorying invertebrate biodiversity: influence of drift-fence length and pitfall trap diameter on spiders. En: The Journal of Arachnology, 33 , pp.681-702.

Bryan, K.M. y Wratten, S.D., 1984. The responses of polyphagous predators to prey spatial heterogeneity: aggregation by carabid and staphylinid beetles to their cereal aphid prey. En: Ecological Entomology, 9, pp.251-259.

Buddle, C.M. y Hammond, H.E.J., 2003. Comparison of ground beetles (Coleoptera: Carabidae) and spiders (Araneae) collected in pan and pitfall traps. En: The Canadian Entomologist, 135, pp.609-611.

Bury, R.B. y Corn, P.S., 1987. Evaluation of pitfall trapping in northwestern forests: Trap arrays with drift fences. En: Journal of Wildlife Management, 51, pp.112-119.

Cameron, K.H. y Leather, S.R., 2012. How good are carabid beetles (Coleoptera, Carabidae) as indicators of invertebrate abundances and order richness? En: Biodiversity and Conservation, 21, pp.763-779.

Capocasale, R.M. y Gudynas, E., 1993. La fauna de opiliones (Arachnida) del criptozoos de Sierra de las Ánimas (Uruguay). En: Aracnología, 19/20, pp.1-15.

Cardinale, B. J., Duffy, E., Gonzalez, A., Hooper, D.U., Perrings, C., Venail, P., Narwani, A., Mace, G.M., Tilman, D., Wardle, D.A., Kinzig, A.P., Daily, G.C., Loreau, M., Grace, J.B., Larigauderie, A., Srivastava, D. y Naeem, S., 2012. Biodiversity loss and its impact on humanity. En: Nature, 486(7401), pp.59-67.

Collins, J.A., Jennings, D.T. y Forsute Jr. H.Y., 1996. Effects of cultural practices on the spiders (Araneae) fauna of Lowbush Blueberry fields in Washington County, Maine. En: The Journal of Arachnology, 24, pp.43-57.

Costa, F.G., Pérez-Miles, F., Gudynas, E., Prandi, L. y Capocasale, R.M., 1991. Ecología de los arácnidos criptozoicos, excepto ácaros, de Sierra de las Ánimas (Uruguay). Ordenes y familias. En: Aracnologia, 13/15, pp1-41.

Curtis, D., 1980. Pitfalls in spider community studies (Arachnida, Araneae). En: The Journal of Arachnology, 8, pp.271-280.

Chen, K-C., Tso, I-M., 2004. Spider diversity on Orchid Island, Taiwan: A comparison between habitats receiving different degrees of human disturbance. En: Zoological Studies, 43(3), pp.598-611.

Churchill, T.B. y Arthur, J.M., 1999. Measuring Spider Richness: Effects of Different Sampling Methods and Spatial and Temporal Scales. En: Journal of Insect Conservation, 3, pp.287-295.

DINAMA, 2010. IV Informe nacional al convenio sobre la diversidad biológica. Montevideo: DINAMA.

Duelli, P. y Obrist, M.K., 1998. In search of the best correlates for local organismal biodiversity in cultivated areas. En: Biodiversity and Conservation, 7, pp.297-309.

Duelli, P. y Obrist, M.K., 2003. Biodiversity indicators: the choice of values and measures. En: Agriculture, Ecosystems and Environment, 98, pp.87-98.

Duffey, E., 2012. Spider Populations and Their Response to Different Habitat Types. En: Arachnology, 15, pp.213-222

Dufrêne, M. y Legendre, P., 1997. Species assemblages and indicator species: the need for a flexible asymmetrical approach. En: Ecological Monographs, 67, pp.345-366.

Ferretti, N., González, A., Pérez-Miles, F., 2014. Identification of priority areas for conservation in Argentina: quantitative biogeography insights from mygalomorph spiders (Araneae: Mygalomorphae). En: Journal of Insect Conservation, 18, pp.1087-1096.

Fournier, E. y Loreau, M., 1999. Effects of new planted hedges on ground-beetle diversity (Coleoptera, Carabidae) in an agricultural landscape. En: Ecography, 22, pp.87-97.

Gaspar, C., Gaston, K.J. y Borges, P.A.V., 2010. Arthropods as surrogates of diversity at differente scales. En: Biological Conservation, 143, pp.1287-1294.

Gerlach, J., Samways, M. y Pryke, J., 2013. Terrestrial invertebrates as bioindicators: an overview of available taxonomic groups. En: Journal of Insect Conservation, 17, pp.831-850.

Ghione, S., Simó, M., Aisenberg, A. y Costa, F.G., 2013. Allocosa brasiliensis (Araneae, Lycosidae) as a bioindicator of coastal sand dunes in Uruguay. En: Arachnology, 16, pp.94-98.

Hendrickx, F., Maelfait, J-P., Van Wingerden, W., Schweiger, O., Speelmans, M., Aviron, S., Augenstein, I., Billeter, R., Bailey, D., Bukacek,j R., Burel, F., Dieköter, T., Dirksen, J., Herzog, F., Liira, J., Roubalova, M., Vandomme, V. y Bugter, R., 2007. How landscape structure, land-use intensity and habitat diversity affect components of total arthropod diversity in agricultural landscapes. En: Journal of Applied Ecology, 44, pp.340-351.

Holopainen, J. K., 1992. Catch and sex ratio of Carabidae (Coleoptera) in pitfall traps filled with ethylene glycol or water. En: Pedobiologia, 36, pp.257-261.

Hore, U. y Uniyal, V.P., 2008. Diversity and composition of spider assemblages in five vegetation types of the Terai Conservation Area, India. En: The Journal of Arachnology, 36, pp.251-258.

Jud, P. y Schmidt-Entling, M.H., 2008. Fluid type, dilution, and bitter agent influence spider preservation in pitfall traps. En: Entomologia Experimentalis et Applicata, 129, pp.356-359.

Koivula, M.J., 2011. Useful model organisms, indicators, or both? Ground beetles (Coleoptera, Carabidae) reflecting environmental conditions. En: Zookeys, 100, pp.287-317.

Kremen, C., Colwell, R.K., Erwin, T.L., Murphy, D.D., Noss, R.F. y Sanjayan, M.A., 1993. Terrestrial arthropod assemblages: their use in conservation planning. En: Conservation Biology, 7, pp.796-808.

Lange, M., Gossner, M.M. y Weisser, W.W., 2011. Effect of pitfall trap type and diameter on vertebrate by-catches and ground beetle (Coleoptera: Carabidae) and spider (Araneae) sampling. En: Methods in Ecology and Evolution, 2, pp.185-190.

Machado, E.O., Laborda, A., Simó, M. y Brescovit, A.D., 2013. Contributions to the taxonomy and distribution of the genus Mesabolivar in southern South America (Araneae: Pholcidae). En: Zootaxa, 3682, pp.401-11.

Machado, E.O., Brescovit, A.D., Candiani, D.F. y Huber, B.A., 2007. Three new species of Mesabolivar (Aranea, Pholcidae) from leaf litter in urban environments in the city of São Paulo, São Paulo, Brazil. En: Iheringia Série Zoologia. 97, pp.168-176.

Matteucci, S. D. y Colma, A., 1982. Metodología para el estudio de la vegetación. Washington: Secretaria General de la Organización de los Estados Americanos. (Serie Biología, Monografía 22).

Moreno, C.E., 2001. Métodos para medir la biodiversidad. Zaragoza: M\&T. (Manuales y Tesis SEA, vol. 1). 
MVOTMA, 2015. ¿Qué es el SNAP? [En línea]. Montevideo: MVOTMA. [Consulta 16 de junio de 2017]. Disponible en: http://www.mvotma.gub.uy/que-es-snap.html

Naeem, S., Chapin, F.S., Costanza, R., Ehrlich, P.R., Golley, F.B., Hooper, D.U., Lawton, J.H., O.Neill, R.V., Mooney, H.A., Sala, O.E., Symstad, A.J. y Tilman, D., 1999. Biodiversity and ecosystem functioning: maintaining natural life support processes. En: Issues in Ecology, 4, pp.1-12.

Niemi, G.J. y McDonald, M.E., 2004. Application of ecological indicators. En: Annual Reviews in Ecology Evolution and Systematics, 35, pp.89-111.

Niemelä, J., Langor, D. y Spence, JR., 1993. Effects of clearcut harvesting on boreal ground-beetle assemblages (Coleoptera: Carabidae) in Western Canada. En: Conservation Biology, 7, pp.551-561.

Pekár, S., 2002. Differential effects of formaldehyde concentration and detergent on the catching efficiency of surface active arthropods by pitfall traps. En: Pedobiologia (Jena), 46, pp.539-547.

Pérez-Miles, F., Simó, M., Toscano-Gadea, C. y Useta, G., 1999. La comunidad de Araneae criptozoicas del Cerro de Montevideo, Uruguay: un ambiente rodeado por urbanización. En: Physis, Sección C, 57, pp.73-87.

R Core Team, 2016. R: A language and environment for statistical computing [En linea]. Vienna: R Foundation for Statistical Computing. [Consulta: 8 de marzo de 2017]. Disponible en: https://www.R-project.org/.

Rainio, J. y Niemelä, J., 2003. Ground beetles (Coleoptera: Carabidae) as bioindicators. En: Biodiversity and Conservation, 12, pp.487-506.

Reichardt, H., 1977. A synopsis of the genera of Neotropical Carabidae (Insecta: Coleoptera). En: Quaestiones Entomologicae, 13, pp.346-493.

Ribera, I. y Foster, G., 1997. El uso de artrópodos como indicadores biológicos. En: Boletín S.E.A., 20, pp.265-276.

Rocha, J.R.M. da, Almeida, J.R., Lins, G.A. y Durval, A., 2010. Insects as indicators of environmental changing and pollution: a review of appropriate species and their monitoring. En: Holos Environment, 10, pp.250-262.

Sauberer, N., Zulka, K.P., Abensperg-Traun, M., Berg, H-M., Bieringer, G., Milasowszky, N., Moser, D., Plutzar, C. Pollheimer, M., Storch, C., Tröstl, R., Zechmeister, H. y Grabherr, G., 2004. Surrogate taxa for biodiversity in agricultural landscapes of eastern Austria. En: Biological Conservation, 117, pp.181-190.
Schirmel, J., Lenze, S., Katzmann, D. y Buchholz, S., 2010. Capture efficiency of pitfall traps is highly affected by sampling interval. En: Entomologia Experimentalis et Applicata, 136, pp.206-210.

Schmidt, M.H., Clough, Y., Schulz, W., Westphalen, A. y Tscharntke, T., 2006. Capture efficiency and preservation attributes of different fluids in pitfall traps. En: The Journal of Arachnology, 34, pp.159-162.

Schmidt, M.H., Roschewitz, I., Thies, C. y Tscharntke, T., 2005. Differential effects of landscape and management on diversity and density of ground-dwelling farmland spiders. En: Journal of Applied Ecology, 42, pp.281-287.

Schowalter, T.D., 2006. Insect ecology: an ecosystem approach. New York: Elsevier.

Simó, M., Laborda, A., Jorge, C. y Castro, M., 2011. Las arañas en agroecosistemas, bioindicadores terrestres de calidad ambiental. En: INNOTEC, 6, pp.51-55.

Skvarla, M.J. y Dowling, A.P.G., 2017. A comparison of trapping techniques (Coleoptera: Carabidae, Buprestidae, Cerambycidae, and Curculionoidea excluding Scolytinae). En: Journal of Insect Science, 17(1), pp.1-28.

Thomas, C.F.G. y Marshall, E.J.P., 1999. Arthropod abundance and diversity in differently vegetated margins of arable fileds. En: Agriculture, Ecosystems and Environment, 72, pp.131-144.

Tonelli, M., Verdú, J.R. y Zunino, M.E., 2017. Effects of grazing intensity and the use of veterinary medical products on dung beetle biodiversity in the submountainous landscape of Central Italy. En: PeerJ, 5, pp.e2780.

Vasconcellos-Neto. J., Romero, G.Q., Santos, A.J. y DippenaarSchoeman, A.S., 2007. Associations of spiders of the genus Peucetia (Oxyopidae) with plants bearing glandular hairs. En: Biotropica, 39, pp.221-226.

Weeks, R.D. y McIntyre, N.E., 1997. A comparison of live versus kill pitfall trapping techniques using various killing agents. En: Entomologia Experimentalis et Applicata, 82, pp.267-273.

Winder, L, Holland, J.M., Perry, J.N., Woolley, C. y Alexander C. J., 2001. The use of barrier-connected pitfall trapping for sampling predatory beetles and spiders. En: Entomologia Experimentalis et Applicata, 98, pp.249-258.

Work, T.T., Buddle, C.M., Korinus, L.M. y Spence, J.R., 2002. Pitfall trap size and capture of three taxa of litter-dwelling arthropods: implications for diversity studies. En: Environmental Entomology, 31(3), pp.438-448. 